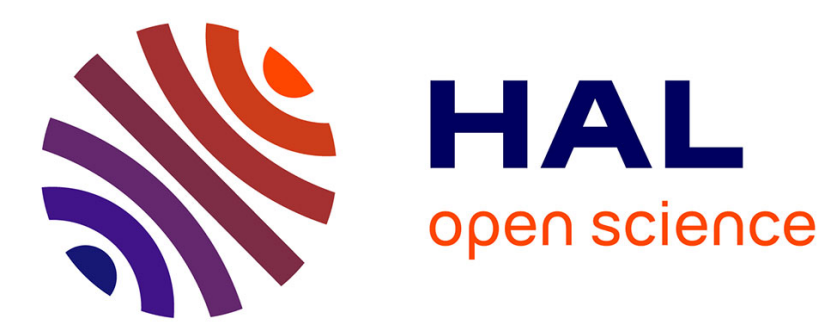

\title{
Le problème du désencastrement
}

Ronan Le Velly

\section{To cite this version:}

Ronan Le Velly. Le problème du désencastrement. Revue du MAUSS, 2007, 29, pp.241-256. hal00452124

\section{HAL Id: hal-00452124 \\ https://hal.science/hal-00452124}

Submitted on 1 Feb 2010

HAL is a multi-disciplinary open access archive for the deposit and dissemination of scientific research documents, whether they are published or not. The documents may come from teaching and research institutions in France or abroad, or from public or private research centers.
L'archive ouverte pluridisciplinaire HAL, est destinée au dépôt et à la diffusion de documents scientifiques de niveau recherche, publiés ou non, émanant des établissements d'enseignement et de recherche français ou étrangers, des laboratoires publics ou privés. 


\section{Le problème du désencastrement}

par Ronan Le Velly

L'encastrement (embeddedness) est une notion clé dans la recherche en sociologie économique, mais une grande ambiguïté demeure quant à la façon dont elle devrait être utilisée ${ }^{1}$. Le titre d'un article de Bernard Barber [1995], «Toutes les économies sont encastrées », est à cet égard assez symptomatique. Quel est le sens d'un tel énoncé ? Pour Barber, il s'agissait d'abord de rappeler, en se référant à Emile Durkheim [1978] et Viviana Zelizer [1978, 1992], combien les facteurs sociaux sont importants dans la formation et le fonctionnement des économies de marché. Mais, en rappelant le caractère toujours encastré de l'économie, Barber souhaitait également s'opposer à un auteur qui fait pourtant figure de référence pour les partisans d'un regard sociologique sur l'économie : Karl Polanyi. Pour aller à l'essentiel et pour rendre hommage au célèbre article de Mark Granovetter [1985], le problème que Barber voyait dans les écrits de Polanyi était celui du désencastrement :

«Polanyi décrit le marché comme "désencastré", les deux autres types d'échanges économiques [la réciprocité et la redistribution] étant plus "encastrés” dans les autres éléments socio-structurels et socioculturels de la société. [...] Notre proposition forte, contrairement à celle de Polanyi, est que toutes les économies sont inévitablement encastrées. [...] Parler de désencastrement du marché détourne notre attention de l'analyse de ce que représente cette interdépendance » [Barber, 1995, p. 400].

Raisonner en terme de désencastrement est-il inadéquat? Et comment comprendre par exemple Jean-Louis Laville lorsqu'il plaide pour un «réencastrement démocratique de l'économie » [Laville, 2003, p. 245] ? Le point de vue que je vais défendre dans cet article est que ces questions gagnent en clarté dès lors que deux notions d'encastrement sont distinguées. Je nommerai ainsi encastrement-étayage la relation à laquelle fait référence Barber [1995] et que mobilisent plus généralement les auteurs de ce qu'il est convenu d'appeler une Nouvelle sociologie économique. Dans cette perspective, le désencastrement est effectivement une absurdité empirique et une impossibilité logique. Sur ce, une telle mise en avant des propriétés de la relation d'encastrement-étayage ne conduit pas forcément à pointer dans les écrits de Polanyi, comme le font Barber mais aussi Granovetter et Fred Block, de l'incohérence ou de la dualité. Au contraire, cet éclairage permet de saisir que lorsque Polanyi fait référence à des processus de désencastrement

\footnotetext{
${ }^{1}$ Cet article reprend une communication proposée lors de la $\mathrm{X}^{\mathrm{e}}$ conférence internationale organisée par le Karl Polanyi Institute à Istanbul en octobre 2005. Je remercie les participants à cette conférence pour leurs encouragements et leurs critiques.
} 
ou de réencastrement, ce n'est pas l'encastrement-étayage qui est en jeu mais ce que j'appellerai une relation d'encastrement-insertion.

\section{L'ENCASTREMENT-ETAYAGE}

L'affirmation de Barber [1995] est à mettre en relation avec le réinvestissement récent des terrains économiques par les sociologues. Ainsi, même si tous les auteurs importants de la nouvelle sociologie économique ${ }^{2}$ ne partagent pas l'utilisation du terme d'encastrement, tous montrent par leurs travaux l'intérêt d'étudier minutieusement les diverses conditions sociales sur lesquelles s'appuie la construction des activités économiques.

Une telle attention est d'abord importante dans une perspective de connaissance empirique. Les premières enquêtes de Granovetter [1973] ou de Zelizer [1978] avaient révélé la primauté du contexte dans le fonctionnement ou l'existence même des marchés et, depuis, de très nombreux travaux ont adopté avec bonheur cette perspective [Dobbin, 2004]. En particulier, étudier les conditions d'encastrement des marchés permet de mieux comprendre pourquoi certains d'entre eux sont plus libéraux ou plus proches du modèle de la théorie économique standard que d'autres. L'ethnographie comparée de plusieurs marchés financiers qu'a menée Mitchel Abolafia [1996] montre que le niveau d'opportunisme, la forme de rationalité ou le degré de concurrence n'ont rien d'une constante. Ils dépendent de l'existence ou non d'outils permettant la transparence de l'information, de différences dans la forme des réseaux et de la nature des règles formelles et morales qui encadrent les comportements spéculatifs. Dans la perspective de la nouvelle sociologie économique, il convient alors d'énoncer qu'un marché très libéral comme le New York Stock Exchange est tout aussi encastré qu'un marché très régulé comme le Chicago Board of Trade: même si ces deux marchés s'appuient sur des conditions d'encastrement très différentes, celles-ci existent autant d'un côté que de l'autre.

La méthodologie de la nouvelle sociologie économique permet de rendre compte de la diversité des situations locales, mais elle vise également à affirmer une théorie de l'action en rupture autant avec celle des économistes qu'avec celle de l'« ancienne » sociologie économique de Talcott Parsons [Granovetter, 1985 ; DiMaggio et Powell, 1991]. Le projet s'inscrit à cet égard dans la série de réorientations théoriques que les sciences sociales ont connues depuis les années 1960 et qui ont pu être qualifiées de «tournant interprétatif », «cognitif » ou «pragmatique » [Thévenot, 1995]. Dès lors que le chercheur refuse une conception mécaniste de l'action, qu'elle consiste en une optimisation rationnelle ou en l'exécution d'un système de normes et de valeurs, il doit être attentif aux opérations par lesquelles les agents interprètent le contexte et 
donnent du sens à leurs actes [Joas, 1999]. En s'appuyant sur les paradoxes de la théorie des jeux, plusieurs articles ont alors montré que, sans les contraintes et les repères associés aux conditions d'encastrement, la coordination entre les agents économiques serait extrêmement problématique, et ce d'autant plus qu'elle implique de s'en remettre à autrui et de lui faire confiance [Orléan, 1994 ; Beckert, 1996]. Au final, dire que les activités économiques sont toujours encastrées signifie que l'engagement dans l'action n'est possible que sur la base de supports sociaux. L'autorégulation, entendue comme vide contextuel, est logiquement incompatible avec un modèle d'action établissant que les agents ont besoin d'interpréter la situation avant d'agir.

En m'inspirant de Caillé [1993], je propose de nommer encastrement-étayage cette première conception de la relation d'encastrement. Il s'agit ici d'une posture méthodologique générale qu'il est possible de relier à la tradition institutionnaliste qui s'est développée aux ÉtatsUnis dans la première moitié $\mathrm{du} \mathrm{Xx}^{\mathrm{e}}$ siècle autour de chercheurs comme John Commons [Bazzoli, 1999 ; Beckert, 2003]. L'action économique est pensée comme étant intimement liée aux conditions institutionnelles qui la permettent autant qu'elles la contraignent. Ces conditions sont le socle indispensable sur lequel se construisent les actions.

De ce point de vue, le vocable de désencastrement n'a effectivement pas de sens s'il suggère l'existence d'une économie sans institutions. À la limite, il reste possible de parler de désencastrement et de réencastrement pour décrire, comme le fait Jens Beckert [1999], un changement dans les conditions d'encastrement. Mais ce terme ne doit à aucun moment évoquer leur disparition et leur réapparition. De la même façon, des niveaux d'encastrement-étayage peuvent être calculés, mais seulement pour traduire en chiffres des différences dans les conditions d'encastrement. Brian Uzzi [1996] évalue ainsi la densité relative de réseaux d'entreprises et montre l'importance de cette variable pour comprendre les phénomènes de faillite. Mais là encore, il n'est pas question d'évaluer un degré d'existence des conditions d'encastrement-étayage, cela n'aurait aucun sens. Les économies sont toujours encastrées-étayées, que cet encastrement prenne la forme de réseaux très denses ou de réseaux atomisés, de règles très contraignantes ou très libérales, etc.

\section{DEUX POLANYI ?}

Je suis donc d'accord avec Barber [1995] lorsqu'il affirme que toutes les économies sont encastrées-étayées. Par contre, la critique qu'il fait à Polanyi me semble particulièrement inadéquate. Il est aujourd'hui clairement établi que Polanyi n'a jamais pensé qu'une économie

\footnotetext{
2 Abolafia, Biggart, Fligstein, Granovetter, Zelizer... - cf. Swedberg [1997].
} 
puisse exister indépendamment des institutions sociales ${ }^{3}$. Ce serait même parfaitement contraire à la méthodologie institutionnaliste qu'il a exposée dans plusieurs textes. Dans L'économie en tant que procès institutionnalisé, Polanyi énonce clairement que l'échange marchand ne doit pas être appréhendé d'une façon différente des autres modalités d'échange. Le marché s'appuie toujours, autant que la réciprocité ou la redistribution, sur des «supports institutionnels déterminés » [Polanyi, 1975, p. 245]. En outre, Polanyi décrit, tout comme le font aujourd'hui les auteurs de la nouvelle sociologie économique, la grande variété des modalités d'organisation des marchés concrets : existence de prix administrés, absence de concurrence en raison d'éléments légaux, géographiques ou coutumiers, situations où seuls les offreurs ou les demandeurs sont actifs... Il existe une «diversité des institutions de marché [qui] fut à une époque récente obscurcie au nom du concept formel du mécanisme offre-demande-prix » [ibid., p. 258].

Le désencastrement dont parle Polanyi dans La Grande Transformation n'est alors certainement pas un désétayage. Au contraire, son analyse insiste longuement sur les changements institutionnels (mouvement des enclosures, fin des poor laws, développement de machines spécialisées...) qui ont permis l'établissement de l'économie libérale du XIX ${ }^{\mathrm{e}}$ siècle. Sa référence à l'autorégulation du marché ne vise pas non plus à présenter une économie fonctionnant dans un vide social. Là encore, c'est tout le contraire. Pour Polanyi, le marché autorégulateur est une utopie, une idée, une institution culturelle qui a nourri le projet politique de la libéralisation économique [Buğra, 2005]. Enfin, la thèse de Polanyi est également que cette utopie n'aurait pu être mise en application sans atteindre à la survie de la société. L'affaiblissement des régulations traditionnelles de l'économie a alors généré la mise en place de nouvelles régulations. Le double mouvement décrit par Polanyi dans La grande transformation est bien, de bout en bout, un processus institutionnalisé.

Aujourd'hui, Granovetter et Block, deux auteurs majeurs de la nouvelle sociologie économique, appréhendent avec justesse l'institutionnalisme des travaux de Polanyi. Dans l'introduction qu'il a rédigée pour la sélection de ses articles traduits en français, Granovetter [2000] explique ainsi qu'il se reconnaît dans le « Polanyi analytique » qui met en avant la pluralité des modes d'allocation des ressources et qui montre la nécessaire régulation des marchés. De façon plus explicite, Block [2003] attribue à Polanyi la découverte de l'always embedded economy et cite même l'article de Barber comme illustration des lectures erronées dont Polanyi a été victime par le passé.

\footnotetext{
${ }^{3}$ Cf. par exemple Gadrey [2000] ou Maucourant [2005].
} 
Pour autant, la réhabilitation de Polanyi par ces deux auteurs n'est pas complète. De nouveau, le problème vient du désencastrement. Dans le même texte, Granovetter exprime son désaccord avec «les affirmations très polémiques que l'on trouve dans [...] La grande transformation, lorsque Polanyi écrit qu'au XIX ${ }^{\mathrm{e}}$ siècle les sociétés sont entrées dans une période radicalement nouvelle où l'économie est devenue désencastrée et qu'elle a alors dominé tous les autres modes d'allocation et tous les autres secteurs » [Granovetter, 2000, p. 39]. L'approbation d'un Polanyi analytique se couple alors avec une prise de distance par rapport au «Polanyi polémique qui surestime l'autonomie du marché » [ibid.]. Il y aurait donc deux Polanyi : le polémiste et l'institutionnaliste... À moins que Polanyi ne se soit transformé de l'un en l'autre. C'est en filigrane la thèse de l'article de Block [2003] sur les conditions de rédaction de La grande transformation. Block explique que les lectures erronées qui ont été faites de cet ouvrage sont la conséquence du caractère incohérent du raisonnement qui y est poursuivi. Polanyi aurait commencé sa rédaction en étant imprégné d'une pensée marxiste hétérodoxe inspirée notamment par Georg Lukacs. C'est dans ce cadre qu'il aurait été amené à décrire les ravages de l'économie autorégulée et le contre-mouvement permettant l'autoprotection de la société. Mais, poursuit Block, au fur et à mesure qu'il décrivait les multiples formes de cette autoprotection, Polanyi « découvrait le concept de l'économie toujours encastrée » [ibid., p. 297] : il découvrait que toute économie a besoin pour fonctionner de règles, de lois et de principes moraux. Pour Block, cette découverte aurait dû conduire Polanyi à reprendre totalement son manuscrit afin de l'expurger des références à l'autorégulation ou au désencastrement. Mais, désireux de publier son ouvrage avant la fin de la guerre pour pouvoir peser sur les débats politiques, ce dernier n'aurait pas pris le temps de le faire :

«Polanyi ne parvient pas à nommer explicitement sa découverte cruciale. Il retourne au contraire à la structure initiale de son raisonnement où l'encastrement de l'économie vient contrer le processus d'autorégulation du marché. Pourtant, la logique même de son raisonnement montre qu'il ne peut jamais exister de système de marché autorégulé. L'idée de contrer son fonctionnement est alors une parfaite absurdité. C'est comme se plaindre qu'une machine à mouvement perpétuel a été abîmée par l'examen d'un scientifique sceptique » [ibid., p. 297].

La thèse de Block est bien documentée, et que Polanyi ait infléchi sa pensée pendant la rédaction de La grande transformation est fort possible. Mais je ne pense pas que la façon dont Block traite du problème du désencastrement soit la bonne. Block, à la différence Barber, voit bien que Polanyi a fait la découverte de «l'économie toujours encastrée ». Mais, tout comme Barber, il pense qu'il y a une totale incompatibilité entre le constat du caractère encastré de l'économie et la description du désencastrement. Pour l'un comme pour l'autre, la cohabitation de ces deux éléments est parfaitement absurde. Dans ces conditions, nous comprenons que, un peu comme 
Granovetter [2000], Block [ibid.] finisse par distinguer deux Polanyi, celui du début et celui de la fin de la rédaction de son ouvrage.

\section{L'ENCASTREMENT-INSERTION}

Alors, y a-t-il deux Polanyi ? Je ne le pense pas. Par contre, je crois qu'une partie du problème pourrait être résolue en exposant le sens que Polanyi attribue à l'idée de désencastrement et en définissant par là même une seconde notion d'encastrement. Plutôt que deux Polanyi, ce sont alors deux relations d'encastrement qui méritent d'être distinguées. Lorsque Polanyi [1983, p. 88] écrit, effectivement sur un ton polémique, qu'avec l'économie de marché « la société est gérée en tant qu'auxiliaire du marché » et que les relations sociales sont désormais «encastrées dans le système économique, il ne pose pas le problème dans les termes de l'encastrement-étayage. Pour comprendre ce qui est en jeu, il nous faut partir de sa description des caractéristiques de ce qu'il nomme l'«économie de marché ».

En comparant cette économie aux autres systèmes économiques, Polanyi [ibid.] signale d'abord le poids exceptionnel qu'y prennent les échanges marchands dans la satisfaction des besoins humains. Suite à l'institution d'un marché pour la terre et pour le travail et à la spécialisation croissante des activités économiques, les individus sont obligés de vendre leur production ou leur force de travail sur le marché afin de survivre. En outre, les marchés institués sont libérés des régulations sociales (publiques, communautaires, professionnelles, religieuses...) propres aux systèmes économiques antérieurs. Une économie de marché est alors une économie où se généralise la recherche du gain individuel et « une économie gouvernée par les prix du marché et par eux seuls » [ibid., p.71]. Polanyi décrit ensuite les conséquences dramatiques de ce mouvement : pollution, désorganisation sociale, vice, perversion sont des mots qu'il lui associe. Ce que Polanyi présente comme un réflexe d'autoprotection de la société ne pouvait alors que s'enclencher dès les années 1860 par l'établissement de régulations non marchandes (règles d'hygiène et de sécurité, assurances contre les accidents du travail...) et se généraliser à partir des années 1930 dans l'avènement de la social-démocratie et du fascisme.

Ce double mouvement est aujourd'hui associé aux termes de désencastrement et de réencastrement. Par exemple, en France, c'est dans cette tradition polanyienne que des auteurs comme Jean-Louis Laville, Bernard Perret ou Guy Roustang utilisent le terme d'encastrement. Ainsi, Roustang [1998] explique que le mouvement de réencastrement décrit par Polanyi est maintenant loin derrière nous. La fin des régimes socialistes, l'intégration de tous les pays à l'économie mondiale de marché et l'affaiblissement des régulations publiques témoignent d'un nouveau désencastrement. Les «impasses de la société de marché » [Roustang et alii, 1996, p. 31] 
rappellent alors celles décrites par Polanyi (désastres écologiques, dérèglements sociaux, appauvrissement culturel) et invitent, selon ces auteurs, à s'engager pour le rétablissement d'une « économie profondément encastrée dans la vie sociale » [ibid., p. 64].

Dans cette perspective, la relation d'encastrement envisagée n'est pas la même que celle décrite dans la première partie. Je propose de nommer cette seconde relation «encastrementinsertion ». La distinction opérée par Alain Caillé [1993] entre pensée du contexte et pensée des ordres résume bien la différence d'approche. Il ne s'agit plus de partir des contextes sur lesquels s'étayent les activités, mais d'évaluer jusqu'à quel point les différents ordres du social sont autonomes-désinsérés et jusqu'à quel point ils sont subordonnés-insérés les uns aux autres. L'approche poursuivie est alors proche des traditions sociologiques qui ont vu dans le développement du capitalisme un processus de différenciation de la sphère économique [ $c f$. Holton, 1992]. Sauf à supposer, associée au désencastrement, une rupture radicale 4 , l'étude comparative des degrés d'encastrement-insertion a alors tout son sens.

En s'inspirant de l'introduction au célèbre recueil Markets in Africa [Bohannan et Dalton, 1965], trois critères peuvent être identifiés pour évaluer ce degré d'encastrement-insertion. Jusqu'à quel point 1) les prix sont-ils le résultat d'une libre confrontation de l'offre et de la demande, 2) la survie matérielle passe-t-elle par la participation au marché et 3) les décisions économiques sontelles guidées par les rémunérations qu'induisent les prix de marché ? Ces trois critères, développés pour la comparaison entre économies, peuvent facilement s'adapter à l'étude des différences de fonctionnement des marchés au sein d'une même économie. Par exemple, le projet du commerce équitable peut être décrit comme celui d'accroître le niveau d'encastrement-insertion du marché de tel produit artisanal ou alimentaire [Raynolds, 2000]. La détermination du prix d'achat aux producteurs prioritairement selon leurs coûts de production, la mobilisation de travail bénévole permettant de s'écarter des impératifs de rentabilité et le choix de travailler avec des producteurs marginalisés même si ceux-ci n'offrent pas les produits les plus performants sont trois exemples, correspondant aux trois critères précédemment identifiés, de principe propre au commerce équitable permettant d'envisager un niveau d'encastrement-insertion du marché plus important que celui qui est associé au marché conventionnel [Le Velly, 2006].

Une dernière illustration de la différence entre l'encastrement-insertion et l'encastrementétayage est possible à la relecture du célèbre article de Granovetter, «Economic action and social structure : the problem of embeddedness » [1985]. De façon intéressante, ces deux relations y sont successivement évoquées, mais, malheureusement, Granovetter n'établit pas de façon explicite leur

\footnotetext{
${ }^{4} C f$. Lacher [1999] pour une lecture intéressante remettant en cause la perspective ici adoptée.
} 
différence de sens. L'article commence ainsi par deux pages d'introduction où Granovetter revient sur les travaux de Polanyi et sur le débat entre anthropologues formalistes et substantialistes. Le point de vue de Granovetter sur ce débat est connu : aucune économie n'est ni parfaitement différenciée ni parfaitement autonome.

«Le niveau d'encastrement du comportement économique est plus faible dans les sociétés non marchandes que ne l'affirment les substantivistes [...] et la "modernisation" a entrâné moins de changement qu'ils ne le pensent. Mais par ailleurs ce niveau a toujours été et continue à être plus substantiel que ne le disent les formalistes et les économistes » [ibid., p. 482-483].

Granovetter parle bien d'un niveau d'encastrement et il ne fait aucun doute que son introduction porte sur l'encastrement-insertion. Mais, dès l'amorce de la première section, les termes du débat changent et s'orientent vers une discussion beaucoup plus méthodologique sur les conceptions sur-socialisées et sous-socialisées de l'action économique. Dans ce contexte, Granovetter utilise de nouveau le terme d'encastrement, mais, cette fois-ci, il n'est plus question de niveaux. Tout le développement de l'article parle de l'encastrement-étayage, de l'indispensable référence au contexte pour comprendre le déroulement des activités économiques. Se succèdent donc dans cet article deux types de débat impliquant deux conceptions différentes de la relation d'encastrement. Mais Granovetter ne semble à aucun moment en avoir clairement conscience. Le compte rendu d'une table ronde permet d'éclairer les raisons de ce mélange [Krippner et alii, 2004]. Granovetter y raconte que, lorsqu'il a rédigé les premières versions de son article, il n'avait pas en mémoire les travaux de Polanyi et les débats entre substantivistes et formalistes. Ce n'est que tardivement qu'il a pris conscience qu'il utilisait le même terme d'encastrement que Polanyi et qu'il a ajouté au début de son texte quelques lignes sur le sujet. Mais, nous l'avons vu, même si le terme retenu était le même, les relations visées étaient différentes. Le message principal de son article a d'ailleurs perdu en clarté, comme en témoignent les fréquentes lectures qui y voient un plaidoyer pour une conception de l'action économique à mi-chemin entre la sur et la soussocialisation, alors que Granovetter revendique, de façon beaucoup plus intéressante, une rupture simultanée avec ces deux théories de l'action tout aussi mécanistes. De façon plus générale, il n'est d'ailleurs pas impossible que nombre d'ambiguïtés qui entourent encore aujourd'hui le terme d'encastrement tiennent pour partie au mélange originel que l'on trouve dans cet article...

\section{DEUX NOTIONS COMPLEMENTAIRES}

L'encastrement-étayage et l'encastrement-insertion renvoient à deux regards différents sur les relations entre l'économique et le social. Mais ces deux regards sont profondément complémentaires. Sur ce point, la référence à Polanyi est de nouveau éclairante. Pour résumer les 
éléments développés dans les deux dernières sections, il est possible de dire que, selon Polanyi, l'économie de marché est 1), comme toutes les économies, une économie institutionnalisée (toujours encastrée-étayée) et 2), plus que toutes les autres, une économie différenciée (au niveau d'encastrement-insertion faible). Présenté ainsi, je ne vois pas en quoi il y aurait un discours incohérent ou inachevé dans La grande transformation. Un tel double regard est d'ailleurs chose commune parmi les sociologues classiques qui ont décrit l'émergence de la société capitaliste. Pour n'en citer qu'un, si Max Weber a exposé de façon exhaustive les conditions institutionnelles qui ont permis l'émergence et le développement du capitalisme ${ }^{5}$, il n'en a pas moins décrit les caractéristiques et les contraintes spécifiques de l'ordre économique qui a résulté de ce mouvement ${ }^{6}$.

Raisonner à la fois sur la forme des conditions d'encastrement-étayage et sur les effets du niveau d'encastrement-insertion permet d'éviter deux formes de réductionnisme. La première, dénoncée avec raison par Zelizer [1992], consiste à perdre de vue les supports institutionnels sur lesquels sont construits les marchés (y compris les plus libéraux) et, en conséquence, à ne voir dans les forces du marché que naturalité, transcendance ou fatalité. La seconde, bien mise en avant par Andrew Sayer [2001], tend à se concentrer tellement sur le caractère socialement institué des marchés qu'elle en oublie les contraintes systémiques qui émergent des ordres marchands et qui échappent à leurs créateurs.

Mobiliser simultanément les deux relations d'encastrement vise à envisager le caractère socialement construit et varié des marchés tout en montrant en quoi les ordres marchands institués imposent des obligations aux agents. Sur ce second point, les auteurs de la nouvelle sociologie économique ont été généralement plutôt discrets. Mais d'autres travaux, particulièrement en anthropologie ou en histoire, ont montré les conséquences du développement d'une économie de plus en plus désencastrée-désinsérée : de nouveaux impératifs de survie, mais aussi de nouvelles opportunités d'enrichissement et de remise en cause des ordres anciens résultent de l'institutionnalisation d'une économie libérale 7 . C'est aussi dans cette perspective que j'ai observé les implications pour les différentes filières du commerce équitable d'une participation plus ou moins forte au cosmos capitaliste. Même si ce niveau de participation est toujours, au moins pour partie, le résultat d'une construction des agents, une participation croissante à l'ordre marchand génère des obligations d'efficacité concurrentielle spécifiques qui tendent à rendre de plus en plus

\footnotetext{
${ }^{5}$ Une bonne vision d'ensemble en est donnée dans la dernière partie de Weber [1991].

6 Pour les références dans l'œuvre de Weber, je me permets de renvoyer à Le Velly [2006].

${ }^{7}$ Cf. par exemple Bourdieu et alii [1963], Haskell et Teichgraeber [1996].
} 
difficile l'établissement du «commerce pas comme les autres» auquel ils aspirent [Le Velly, 2006].

Le double regard envisagé par cet article peut enfin faire apparaître des déterminations circulaires. Stephen Kalberg [2002] montre l'importance dans les écrits de Weber de ce qu'il nomme un «modèle de développement du marché libre » où l'expansion du marché de rationalité formelle contient en elle-même une « force motrice » capable d'affaiblir les régulations qui lui sont contraires. De même, lorsque Émile Durkheim [1978] étudie les conditions et les causes de la division du travail, les relations qu'il établit entre, d'un côté, le développement de la division du travail et, de l'autre côté, le développement du droit des contrats et la condensation de la société sont des relations de renforcement mutuel. C'est aussi cette logique d'autorenforcement que Joyce Appleby [1978] met parfaitement en avant lorsqu'elle décrit le développement d'une culture du marché (croyances dans la légitimité de la poursuite de l'intérêt personnel, dans l'autonomie des phénomènes économiques...) comme à la fois une précondition et un résultat de l'émergence de l'économie libérale anglaise. Pour autant, une telle circularité cumulative n'est pas non plus systématique et il convient d'observer des situations où les contraintes qui émergent des ordres marchands entrent en conflit avec les conditions d'encastrement sur lesquelles s'appuient ces ordres. Les effets désastreux de l'utopie du marché autorégulateur exposés par Polanyi [1983] renvoient à cette seconde classe de phénomènes, et les interrogations actuelles sur les effets déstructurants des stratégies de libéralisation et de la mondialisation libérale vont également dans ce sens [Stiglitz, 2002].

\section{CONCLUSION}

Dans un entretien récent, Beckert, un des sociologues qui le mieux décrit le contenu de la relation d'encastrement-étayage, regrettait que les travaux de la Nouvelle sociologie économique se soient restreints à l'exploration de cette seule dimension des rapports entre marché et société :

« Je ne suis pas satisfait de l'étroitesse avec laquelle le concept d'encastrement est utilisé par la Nouvelle sociologie économique. Pour Polanyi, l'encastrement était un concept dirigé de façon critique à l'encontre du modèle de marché libéral. Il pointait du doigt le besoin d'une intervention pour réguler le marché et compenser les effets socialement problématiques du système marchand. Dans la Nouvelle sociologie économique, le concept se concentre sur les structures sociales des marchés sans traiter de leurs conséquences pour la société d'une façon plus large, incluant leurs effets sur l'ordre social, la justice et l'égalité. (...) En cela, la Nouvelle sociologie économique ne relève pas le défi posé par Karl Polanyi » [Beckert, 2006, p. 37].

Il paraît effectivement légitime de s'interroger sur les implications politiques d'une perspective théorique qui, dans la lignée de Block [2003], revendiquerait de ne s'intéresser qu'à la 
relation d'encastrement-étayage. Le point de vue constructiviste qu'adopte la notion d'encastrement-étayage est riche de sens politique en ce qu'il rappelle que les marchés ne fonctionnent jamais que selon les conditions institutionnelles qui les encadrent, et donc selon les choix politiques qui président à leur construction. Mais une telle démonstration ne doit pas s'arrêter en si bon chemin, et je ne vois pas de bonnes raisons pour ne pas être également attentif aux conséquences des différentes modalités de régulation économique et aux dynamiques institutionnelles qu'elles engendrent. Pour le dire autrement: si la perspective constructiviste conduit à énoncer que, dans le capitalisme, toutes les formes de marché sont possibles, elle ne devrait pas, sous peine d'adopter une vision bien angélique tant du marché que du capitalisme, négliger les obligations qui émergent des ordres marchands et qui à leur tour contraignent la construction des marchés.

\section{BIBLIOGRAPHIE}

Abolafia M. Y., 1996, Making Markets : Opportunism and Restraint on Wall Street, Harvard University Press, Cambridge.

APPLeBY J., 1978, Economic Thought and Ideology in Seventeenth-Century England, Princeton University Press, Princeton.

BARBER B., 1995, «All economics are "embedded" : the career of a concept and beyond », Social Research, vol. 62, $\mathrm{n}^{\circ}$ 2, p. 387-414.

BAzzoli L., 1999, L’Économie politique de John R. Commons. Essai sur l'institutionnalisme en sciences sociales, L'Harmattan, «Études d'économie politique », Paris.

BECKERT J., 1996, «What is sociological about economic sociology? Uncertainty and the embeddedness of economic action », Theory and Society, vol. 25, n 6, p. 803-840.

- 1999, «Agency, entrepreneurs and institutional change. The role of strategic choice and institutionalised practices in organizations », Organization Studies, vol. 20, n 5, p. 777-799.

- 2003, «Economic action and embeddedness : how shall we conceptualize economic action? », Journal of Economic Issues, vol. 37, $\mathrm{n}^{\circ} 3$, p. 769-787.

- 2006, «Ten questions about economic sociology », Economic sociology, the European electronic newsletter, vol. 7, $\mathrm{n}^{\circ} 3$, p. 34-39.

Biggart N. W., 1988, Charismatic Capitalism. Direct Selling Organization in America, The University of Chicago Press, Chicago.

BLOCK F., 2003, « Karl Polanyi and the writing of "The Great Transformation" », Theory and Society, vol. 32, n) 3, p. 275-306. 
Bohannan P. et Dalton G. (sous la dir. de), 1965, Markets in Africa, Eight Subsistence Economy in Transition, Anchor books, New York.

Bourdieu P., Darbel A., Rivet J.-P. et Seibel C., 1963, Travail et travailleurs en Algérie, Mouton-MSH, La Haye-Paris.

BugRA A., 2005, «Karl Polanyi et la séparation institutionnelle entre politique et économie », Raisons politiques, $\mathrm{n}^{\circ} 20$, p. 27-56.

CAILle A., 1993, La Démission des clercs. La crise des sciences sociales et l'oubli du politique, La Découverte, « Armilliaire », Paris.

DiMaggio P. et Powell W., 1991, «Introduction », in DiMaggio P. et Powell W. (sous la dir. de), The New Institutionalism in Organizational Analysis, The University of Chicago Press, Chicago.

Dobbin F., 2004, The new economic sociology, a reader, Princeton University Press, Princeton.

DURKHEIM É., [1893] 1978, De la division du travail social, PUF, Paris.

GAdREY J., 2000, Nouvelle Économie, nouveau mythe ?, Flammarion, « Essais », Paris.

Granovetter M., 1973, « The strength of weak ties », American jJournal of Sociology, vol. 78, n 6, p. 1360-1380 (traduit en français dans Granovetter, 2000).

- 1985, «Economic action and social structure : the problem of embeddedness », American Journal of Sociology, vol. 91, n³ 3, p. $481-510$ (traduit en français dans Granovetter, 2000).

- 2000, Le Marché autrement, Desclée De Brouwer, « Sociologie économique », Paris.

Haskell T. L. et Teichgraeber R. F. (sous la dir. de), 1996, The Culture of the Market. Historical Essays, Cambridge University Press, New York.

Holton R., 1992, Economy and Society, Routledge, Londres.

JOAS H., 1999, La Créativité de l'agir, Éditions du Cerf, « Passages », Paris.

KAlberg S., 2002, 1994, La Sociologie historique comparative de Max Weber, La Découverte/MAUSS, Paris.

KripPner G., Granovetter M., Block F. et Biggart N., 2004, «Polanyi symposium : a conversation on embeddedness », Socio-Economic Review, vol. 2, n 1, p. 109-135.

LACHER H., 1999, « The politics of the market : re-reading Karl Polanyi », Global Society, vol. $13, n^{\circ} 3$, p. 313-326.

Laville J.-L., 2003, «Avec Mauss et Polanyi, vers une théorie de l'économie plurielle », Revue du MAUSS semestrielle, $\mathrm{n}^{\circ} 21$, p. 237-249.

LE VELly R., 2006, «Le commerce équitable : des échanges marchands contre et dans le marché », Revue française de sociologie, vol. 47, n², p. 319-340. 
Maucourant J., 2005, Avez-vous lu Polanyi ?, La Dispute, Paris.

ORLEAN A., 1994, «Introduction. Vers un modèle général de la coordination économique par les conventions », in ORLEAN A. (sous la dir. de), Analyse économique des conventions, PUF, Paris.

POLANYI K., [1957] 1975, «L'économie en tant que procès institutionnalisé », in POLANYI K., Arensberg C, Pearson H. (sous la dir. de), Les Systèmes économiques dans l'histoire et la théorie, Larousse Université, Paris.

- [1944] 1983, La Grande Transformation. Aux origines politiques et économiques de notre temps, Gallimard, « NRF », Paris.

RAYNOLDS L. T., 2000, «Re-embedding global agriculture : the international organic and fair trade movements », Agriculture and Human Values, ${ }^{\circ}$ 17, p. 297-309.

Roustang G., 1998, «"Grande transformation” ou "alliage sans formule précise” ? », in Servet J.-M., Maucourant J., Tiran A. (sous la dir. de), La Modernité de Karl Polanyi, L’Harmattan, « Logiques sociales », Paris.

Roustang G., Laville J.-L., Eme B., Mothe D. et Perret B., 1996, Vers un nouveau contrat social, Desclée de Brouwer, «Sociologie économique », Paris.

SAYER A., 2001, «For a critical cultural political economy », Antipode, vol. 33, n 4, p. 687-708.

Stiglitz J., 2002, La Grande Désillusion, Fayard, Paris.

SWEDBERG R., 1997, «Vers une nouvelle sociologie économique : bilan et perspectives », Cahiers internationaux de sociologie, CIII, p. 237-263.

THEVENOT L., 1995, «Rationalité ou normes sociales : une opposition dépassée ? », in Gerard-VAlet L., PASSERon J.-C. (sous la dir. de), Le Modèle et l'enquête. Les usages du principe de rationalité dans les sciences sociales, Éditions de l’EHESS, Paris.

UzZI B., 1996, «The sources and consequences of embeddedness for the economic performance of organisations : the network effect », American Sociological Review, vol. 61, $\mathrm{n}^{\circ} 4$, p. 674-698.

WEBER M., [1923] 1991, Histoire économique. Esquisse d'une histoire universelle de l'économie et de la société, Gallimard, « NRF », Paris.

ZELIZER V. A., 1978, «Human values and the market : the case of life insurance and death in 19th century America », American Journal of Sociology, n 84, p. 591-610.

- 1992, «Repenser le marché : la construction sociale du marché aux bébés aux États Unis, 1870-1930 », Actes de la recherche en sciences sociales, $n^{\circ} 94$, p. 3-26. 